\title{
When cows lie down
}

Start with 70-odd cows: some pregnant, some kept indoors, others wintering outdoors. Next, fix delicate motion sensors to their legs so that you can record precisely when they stand up and when they lie down. Then wait a while to collect ample data recordings of, say, at least 50,000 episodes of lying down and standing back up. What you have then is the raw material for a research report in Applied Animal Behaviour Science, under the title "Are cows more likely to lie down the longer they stand?"

This curious paper of a few years back, by Bert Tolkamp and colleagues, reports something interesting about how these cows switch between the two states of lying and standing (Appl. Anim. Behav. Sci. 124, 1-10; 2010); yes, this is science. The understanding gained may well improve farming practice, as well as animal welfare. But it also touches on some deeper mysteries.

When a cow is standing, the data show that the likelihood of its deciding to lie down at any moment remains constant through time. That is, a cow that has been standing for two hours isn't any more likely to lie down in the next five minutes than one that stood up only a few minutes ago. To say it like a physicist, it seems that a cow 'decays' from standing to lying in much the way an unstable particle decays - with constant probability per unit time. There is no 'memory' in the process, or evidence of any kind of physiological control that would make an animal seek to lie down more strongly after a sufficient time standing. In contrast, the lying cow does become ever more likely to stand as time passes.

I doubt anyone will be surprised to hear that this bit of work was chosen as one of the winners of the 2013 Ig Nobel Prizes, given out for research that seems a little pointless or silly, but actually isn't. Possibly, what Tolkamp and colleagues found may not be unique to cows, as there are some intriguing links to patterns of switching behaviour elsewhere in biology.

In a recent study reported in Nature, biologist Thomas Norman and colleagues essentially repeated this experiment, only using the bacterium Bacillus subtilis rather than cows (Nature 503, 481-486; 2013). This bacterium has a similar switching behaviour, fluctuating between a motile state where it swims about and a sessile or resting state, in which it stays put and often links up with other bacteria. The former state is akin to the standing cow; the latter state to the lying cow. Using fluorescent

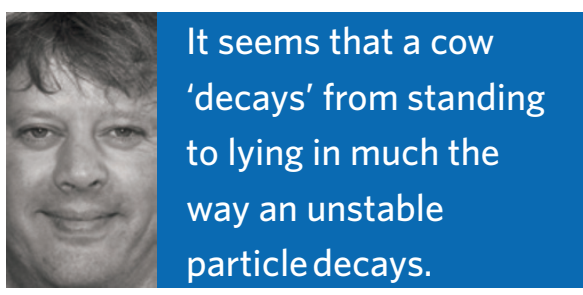

proteins, Norman et al. were able to monitor switching in a population of these bacteria, and to do so under special conditions in which they could eliminate all possible external signals that might induce switching.

Hence, they observed switching as governed only by autonomous internal controls. (This is, in a way, analogous to the controlled conditions for the cow experiments, which tried to eliminate signals and disturbances that might drive standing or lying transitions.) This autonomous switching pattern, Norman and colleagues found, is identical to that of the cows: motile bacteria enter the sessile state in a purely random fashion, with a fixed probability per unit time. Once sessile, however, there's a fairly tight schedule for returning to the motile state - it happens after roughly eight generations of a single cell.

It's certainly interesting to find that creatures differing in volume by a factor of a trillion or so might - in one simple aspect of their behaviour under controlled conditions - act more or less identically. What's the explanation? In the case of the bacteria, Norman and colleagues argue that the dynamic does make sense in the context of bacterial social behaviour.

Biologists know that bacteria such as B. subtilis will, under many conditions, spontaneously aggregate into long chains, which then come together to form a biofilm - a protective matrix that can enable a bacterium to survive for long periods in difficult environmental conditions. This transition is ordinarily driven by environmental signals, such as a lack of food sources. To maintain this mechanism in a sensitive condition, however, it seems that bacteria perpetually create 'trial' chains by falling temporarily into the sessile state. These trial chains act as seeds, the growth of which can be reinforced by external signals when appropriate. Norman et al. suggest that the tight timing of chain-breaking transitions back into the motile state helps to tune the population of existing seed chains in the right way.
These biologists (in earlier work) were also able to go one impressive step further, actually identifying a simple non-linear genetic feedback circuit that controls this autonomous switching behaviour in $B$. subtilis. In this case, the circuit involves just three genes and their respective proteins. The two states, motile and sessile, correspond to high expression of two respective proteins, SinR and SlrR. These proteins, when expressed, act through different mechanisms to keep the expression of their counterpart low. Hence, there's a two-state switch. A further protein, SinI, can act to drive the switch into the sessile state if triggered by environmental signals. Stochastic fluctuations can also drive the same transition in the absence of any external signal.

Now, what about the cows? If going into the sessile state allows bacteria to aggregate, this isn't at all the case for cows lying down. Cows readily lie together in shady grass, and at other times stand together in parallel alignment basking in the Sun's rays. As Tolkamp et al. point out, however, there is one fairly obvious possible explanation for why cows grow more likely to stand after lying for a time. Cows only eat when standing, so a lying cow is also an increasingly hungry cow - and therefore more likely to stand up to get some food. The detailed pattern of standing behaviour seems to mesh quite well with this idea.

So there may be no real functional similarity in this behaviour between cows and bacteria; it serves different ends in the two cases. Of course, the behaviour of a cow is far richer than that of any bacterium, and control in multicellular organisms isn't anything like that in a single-celled one. The similarity may only be accidental, the consequence of some fairly generic mechanism for switching control.

As Norman and colleagues have been able to identify the control circuit in the bacteria, it remains to be seen if anything similar is active in the control machinery of cows. Although no doubt much more complex, the genetic circuitry might well reveal some kernel of similarity. Or not. Might there be vestiges of such simple control circuits operating in multicellular organisms, including higher mammals, and perhaps even humans? I wouldn't bet against it.

\section{MARK BUCHANAN}

